Virginia Commonwealth University VCU Scholars Compass

2012

\title{
Bevacizumab Added to Neoadjuvant Chemotherapy for Breast Cancer
}

Harry D. Bear

Virginia Commonwealth University, hdbear@vcu.edu

Gong Tang

University of Pittsburgh

Priya Rastogi

University of Pittsburgh

See next page for additional authors

Follow this and additional works at: http://scholarscompass.vcu.edu/massey_pubs

Part of the Medicine and Health Sciences Commons

From The New England Journal of Medicine, Bear, H. D., Tang, G., Rastogi, P. et al., Bevacizumab Added to Neoadjuvant Chemotherapy for Breast Cancer, Vol. 366, Page 310, Copyright (C) 2012 Massachusetts Medical Society.

\section{Downloaded from}

http://scholarscompass.vcu.edu/massey_pubs/8

This Article is brought to you for free and open access by the Massey Cancer Center at VCU Scholars Compass. It has been accepted for inclusion in Massey Cancer Center Publications by an authorized administrator of VCU Scholars Compass. For more information, please contact libcompass@vcu.edu. 


\section{Authors}

Harry D. Bear, Gong Tang, Priya Rastogi, Charles E. Geyer Jr., Andre Robidoux, James N. Atkins, Luis BaezDiaz, Adam M. Brufsky, Rita S. Mehta, Louis Fehrenbacher, James A. Young, Francis M. Senecal, Rakesh Gaur, Richard G. Margolese, Paul T. Adams, Howard M. Gross, Joseph P. Costantino, Sandra M. Swain, Eleftherios P. Mamounas, and Norman Wolmark 


\title{
Bevacizumab Added to Neoadjuvant Chemotherapy for Breast Cancer
}

\author{
Harry D. Bear, M.D., Ph.D., Gong Tang, Ph.D., Priya Rastogi, M.D., \\ Charles E. Geyer, Jr., M.D., André Robidoux, M.D., James N. Atkins, M.D., \\ Luis Baez-Diaz, M.D., Adam M. Brufsky, M.D., Ph.D., Rita S. Mehta, M.D., \\ Louis Fehrenbacher, M.D., James A. Young, M.D., Francis M. Senecal, M.D., \\ Rakesh Gaur, M.D., M.P.H., Richard G. Margolese, M.D., C.M., Paul T. Adams, M.D., \\ Howard M. Gross, M.D., Joseph P. Costantino, Dr.P.H., Sandra M. Swain, M.D., \\ Eleftherios P. Mamounas, M.D., and Norman Wolmark, M.D.
}

From the National Surgical Adjuvant Breast and Bowel Project (NSABP) (H.D.B., G.T., P.R., C.E.G., A.R., J.N.A., L.B.-D., A.M.B., R.S.M., L.F., J.A.Y., F.M.S., R.G., R.G.M., P.T.A., H.M.G., J.P.C., S.M.S., E.P.M., N.W.); the NSABP Biostatistical Center and the Department of Biostatistics, University of Pittsburgh Graduate School of Public Health (G.T., J.P.C.); University of Pittsburgh Cancer Institute, University of Pittsburgh (P.R., A.M.B.); and Allegheny General Hospital (N.W.) - all in Pittsburgh; Medical College of Virginia School of Medicine and the Massey Cancer Center, Virginia Commonwealth University, Richmond (H.D.B.); University of Texas Southwestern Medical Center, Dallas (C.E.G.); Centre Hospitalier de I'Université de Montréal (A.R.) and Jewish General Hospital, McGill University (R.G.M.) - both in Montreal; South East Cancer Control Consortium Community Clinical Oncology Program (CCOP), Goldsboro, NC (J.N.A.); Minority-Based CCOP, San Juan, Puerto Rico (L.B.-D.); University of California at Irvine, Orange (R.S.M.); Kaiser Permanente Oncology Clinical Trials, Northern California, Vallejo, CA (L.F.); Colorado Cancer Research Program, Colorado Springs (J.A.Y.); Northwest Medical Specialties, Tacoma, WA (F.M.S.); CCOP, Kansas City, MO (R.G.); Genesys Regional Medical Center, Grand Blanc, MI (P.T.A.); CCOP, Dayton (H.M.G.), and Aultman Hospital Cancer Center, Canton (E.P.M.) - both in Ohio; and Washington Cancer Institute, Medstar Washington Hospital Center, Washington, DC (S.M.S.). Address reprint requests to Dr. Bear at Box 980011 , Division of Surgical Oncology, VCUHS, Richmond, VA 23298-0011, or at hdbear@vcu.edu.

N Engl J Med 2012;366:310-20.

Copyright @ 2012 Massachusetts Medical Society.

\section{ABSTRACT}

\section{BACKGROUND}

Bevacizumab and the antimetabolites capecitabine and gemcitabine have been shown to improve outcomes when added to taxanes in patients with metastatic breast cancer. The primary aims of this trial were to determine whether the addition of capecitabine or gemcitabine to neoadjuvant chemotherapy with docetaxel, followed by doxorubicin plus cyclophosphamide, would increase the rates of pathological complete response in the breast in women with operable, human epidermal growth factor receptor 2 (HER2)-negative breast cancer and whether adding bevacizumab to these chemotherapy regimens would increase the rates of pathological complete response.

METHODS

We randomly assigned 1206 patients to receive neoadjuvant therapy consisting of docetaxel (100 mg per square meter of body-surface area on day 1), docetaxel (75 mg per square meter on day 1) plus capecitabine ( $825 \mathrm{mg}$ per square meter twice a day on days 1 to 14), or docetaxel (75 mg per square meter on day 1) plus gemcitabine (1000 mg per square meter on days 1 and 8) for four cycles, with all regimens followed by treatment with doxorubicin-cyclophosphamide for four cycles. Patients were also randomly assigned to receive or not to receive bevacizumab (15 mg per kilogram of body weight) for the first six cycles of chemotherapy.

\section{RESULTS}

The addition of capecitabine or gemcitabine to docetaxel therapy, as compared with docetaxel therapy alone, did not significantly increase the rate of pathological complete response (29.7\% and $31.8 \%$, respectively, vs. $32.7 \%$; $\mathrm{P}=0.69)$. Both capecitabine and gemcitabine were associated with increased toxic effects - specifically, the hand-foot syndrome, mucositis, and neutropenia. The addition of bevacizumab significantly increased the rate of pathological complete response $(28.2 \%$ without bevacizumab vs. $34.5 \%$ with bevacizumab, $\mathrm{P}=0.02$ ). The effect of bevacizumab on the rate of pathological complete response was not the same in the hormone-receptor-positive and hormone-receptor-negative subgroups. The addition of bevacizumab increased the rates of hypertension, left ventricular systolic dysfunction, the hand-foot syndrome, and mucositis.

\section{CONCLUSIONS}

The addition of bevacizumab to neoadjuvant chemotherapy significantly increased the rate of pathological complete response, which was the primary end point of this study. (Funded by the National Cancer Institute and others; ClinicalTrials.gov number, NCT00408408.) 
$\mathrm{N}$

EOADJUVANT CHEMOTHERAPY HAS BEcome established as a reasonable alternative to adjuvant chemotherapy for operable breast cancer, since it can increase the rates of breast-conserving surgery ${ }^{1-3}$ and decrease the need for complete axillary lymph-node dissection. .-6 $^{4-6}$ Neoadjuvant chemotherapy also offers the potential for rapidly testing regimens that may improve response rates and therefore may be likely to improve the outcomes in patients. Although alterations in neoadjuvant chemotherapy that increase the rates of pathological complete response may not necessarily improve survival, ${ }^{5,7}$ the results of the National Surgical Adjuvant Breast and Bowel Project (NSABP) B-27 trial (ClinicalTrials.gov number, NCT00002707) of neoadjuvant therapy were concordant with those of randomized trials of adjuvant therapy that showed improved outcomes with the addition of taxanes. ${ }^{3,8-11}$ Evaluation of responses of tumors to neoadjuvant therapy in patients who have not previously been exposed to systemic therapies could be a more useful strategy for determining the drugs or regimens that are worth testing in trials of adjuvant therapy than a strategy of using results from studies involving patients with metastatic disease. Furthermore, the neoadjuvant setting allows for the collection of tissues that can be used to identify predictors of treatment response and that can inform the design of future trials of adjuvant therapy.

Bevacizumab (Avastin, Genentech), an antiangiogenic monoclonal antibody against vascular endothelial growth factor (VEGF) A, and the antimetabolites capecitabine and gemcitabine have been shown to improve the outcomes when added to taxanes in patients with metastatic breast cancer. Bevacizumab added to chemotherapy increased progression-free survival and the rates of response, but not overall survival, in prospective, randomized trials involving patients with metastatic breast cancer. ${ }^{12-14}$ The addition of capecitabine to the taxane docetaxel in patients with locally advanced or metastatic disease increased the rates of objective response and significantly prolonged the median time to progression and the median overall survival. ${ }^{15}$ In a phase 3 , randomized trial, gemcitabine added to paclitaxel significantly increased the rates of response, the median time to progression, and the median overall survival. ${ }^{16}$ With that background, we sought to determine whether adding capecitabine or gemcitabine to docetaxel, followed by the anthracycline doxorubicin plus cyclophosphamide, would improve the outcomes in patients with operable, human epidermal growth factor receptor 2 (HER2)-negative breast cancer and to determine the effect of the addition of bevacizumab to these neoadjuvant chemotherapy regimens.

\section{METHODS}

\section{PATIENT ELIGIBILITY AND ENTRY PROCEDURES}

Women with primary operable HER2-negative breast cancer diagnosed by means of a core needle biopsy were eligible for participation in the study. Patients were required to have a palpable primary tumor at least $2.0 \mathrm{~cm}$ in diameter in the breast, as assessed by physical examination, and to be classified as having tumor stage T1c to T3, nodal stage N0 to N2a, and metastasis stage M0. Other key eligibility criteria were an Eastern Cooperative Oncology Group (ECOG) performance status of 0 or 1 (with 0 indicating that the patient is fully active and able to carry on all predisease activities without restriction and 1 indicating that the patient is restricted in physically strenuous activity but is ambulatory and able to carry out work of a light or sedentary nature, such as light housework or office work) and normal left ventricular ejection fraction, determined by multiple-gated acquisition scanning or echocardiography within 3 months before study entry. Before random assignment, patients were required to have core-biopsy material harvested for correlative science studies.

The assignment to treatment groups was balanced according to age at entry ( $\leq 49$ or $\geq 50$ years), clinical tumor size $(2.0$ to $4 \mathrm{~cm}$ or $\geq 4.1 \mathrm{~cm}$ ), hormone-receptor status (estrogen-receptor-positive, progesterone-receptor-positive, or both vs. estrogenreceptor-negative and progesterone-receptor-negative), and clinical nodal status (negative vs. positive). Randomization was performed within these strata, with the use of a biased-coin approach to ensure balanced treatment assignments within an institution.

\section{STUDY OVERSIGHT}

The protocol was approved by the central institutional review board of the National Cancer Institute (NCI) and by the human investigations committee or institutional review board at each participating site, each of which has approval for human subjects research from the Department of Health and Human Services. Written informed consent was obtained from all participants. The first author wrote the first draft of the manuscript, 
and all the authors contributed to subsequent drafts and made the decision to submit the manuscript for publication. A group of NSABP authors ensured the fidelity of the study to the protocol. F. Hoffmann-La Roche, Genentech USA, and Eli Lilly provided partial support for this trial and were provided an opportunity to review the manuscript before submission for publication. The NSABP restricts the access of sponsors to outcomes data until the time of publication. The study had no additional commercial support, and no person other than the authors contributed to the content of the manuscript. The protocol, including the statistical analysis plan, is available with the full text of this article at NEJM.org.

\section{TREATMENT}

Eligible patients were randomly assigned to one of three neoadjuvant chemotherapy regimens: four cycles of docetaxel (100 mg per square meter of body-surface area, administered intravenously on day 1 of the cycle) every 3 weeks, followed by four cycles of doxorubicin-cyclophosphamide (60 $\mathrm{mg}$ and $600 \mathrm{mg}$ per square meter, respectively, administered intravenously every 3 weeks) (docetaxel group); capecitabine ( $825 \mathrm{mg}$ per square meter, administered orally twice a day on days 1 through 14) added to docetaxel (75 mg per square meter, administered intravenously on day 1), followed by doxorubicin-cyclophosphamide (docetaxel-capecitabine group); or gemcitabine (1000 mg per square meter, administered intravenously on days 1 and 8) added to docetaxel (75 mg per square meter, administered intravenously on day 1), followed by doxorubicin-cyclophosphamide (docetaxel-gemcitabine group) (see Fig. S1 in the Supplementary Appendix, available at NEJM.org). Half the patients were also randomly assigned to receive bevacizumab (15 mg per kilogram of body weight, administered intravenously, every 3 weeks) with each of the first six cycles of chemotherapy and for 10 additional doses every 3 weeks postoperatively (Fig. S1 in the Supplementary Appendix). Bevacizumab was stopped after cycle 6 for a washout before surgery in order to reduce the risk of surgical complications.

Patients who were considered to be candidates for breast-conserving surgery were to have the primary tumor site marked (with clips or tattoos) before the initiation of chemotherapy. Patients underwent surgery after they had recovered from chemotherapy, the final tumor assessments had been performed, and cardiac function had been evaluated. For patients receiving bevacizumab who underwent all four cycles of doxorubicincyclophosphamide, surgery was performed at least 9 weeks after the last dose of bevacizumab. If chemotherapy was stopped before completion of the planned therapy, surgery was performed at least 4 weeks, and preferably 6 weeks, after the last dose of bevacizumab. The type of surgery that was performed was left to the discretion of the patient and surgeon. For patients undergoing breast reconstruction, tissue expansion could not be performed within the 2 weeks before the first postoperative dose of bevacizumab. Expansion or any surgical procedure (e.g., exchanging tissue expanders for permanent implants) was prohibited throughout the course of bevacizumab therapy and a minimum period of 6 weeks after the last dose of bevacizumab. Post-lumpectomy breast radiation therapy was required after breast-conserving surgery. Partial breast irradiation was not permitted. Decisions regarding regional-node irradiation and post-mastectomy irradiation were made at the discretion of the patient's physician. Patients with estrogen-receptor-positive or progesterone-receptor-positive tumors received endocrine therapy for a minimum of 5 years after the completion of neoadjuvant chemotherapy and surgery. The selection of endocrine agents was left to the discretion of the physicians.

\section{END POINTS}

The primary end point was the rate of pathological complete response in the breast. Secondary end points included the rate of pathological complete response in the breast and nodes; clinical complete responses after the docetaxel-based portion of the chemotherapy program had been completed; clinical complete responses after completion of the entire sequential chemotherapy program; the percentage of patients with cardiac events, defined as New York Heart Association (NYHA) class III or IV congestive heart failure; and toxic effects, including cardiac events other than congestive heart failure.

\section{ASSESSMENTS OF TUMOR RESPONSE AND ADVERSE EVENTS}

The assessment of tumor response was based on modifications of the criteria proposed by the Response Evaluation Criteria in Solid Tumors Group. ${ }^{17}$ A pathological complete response in the breast 
was defined as the absence of histologic evidence of invasive tumor cells in the surgical breast specimen. A pathological complete response in the breast and nodes was defined as the absence of histologic evidence of invasive tumor cells in the surgical breast specimen, axillary nodes, and nonaxillary sentinel nodes identified after neoadjuvant chemotherapy. Disease progression was defined as the unequivocal progression of existing target or nontarget lesions; the appearance of one or more new lesions in the breast, regional lymph nodes, or distant sites; or the appearance of inflammatory carcinoma on clinical examination. Adverse events were graded according to the NCI Common Terminology Criteria for Adverse Events, version 3.0 (http://ctep.cancer.gov/protocolDevelopment/ electronic_applications/docs/ctcaev3.pdf).

\section{STATISTICAL ANALYSIS}

There were two primary hypotheses: that the addition of capecitabine or gemcitabine would improve the rate of pathological complete response in the breast, and that the addition of bevacizumab would improve the rate of pathological complete response in the breast. The estimation of the sample size was based on the comparison among chemotherapy regimens. Assuming that the rate of pathological complete response in the docetaxel group would be $26 \%$, we estimated that we would need to enroll 400 patients in each of the three docetaxel-based groups for the study to have $80 \%$ power to detect a significantly different rate of $36 \%$ for pathological complete response in either the capecitabine group or the gemcitabine group, with a type I error rate of 0.05 .

The analyses of end-point data are based on information gathered as of June 30, 2011. The maximum of two standardized pairwise differences in the rates of pathological complete response between the docetaxel group and the other two groups, with or without bevacizumab, was used for testing the improvement in the outcome with the addition of capecitabine or gemcitabine. ${ }^{18}$ The critical value for a 0.05 significance level is 2.21 , which was calculated from 10,000 simulations with adjustment for multiple comparisons. ${ }^{18}$ The Pearson chi-square test with continuity adjustment $^{19}$ was used to assess the association between treatment and response variables. The Breslow-Day test was performed to assess the homogeneity of the odds ratios across randomization strata and histologic grades. ${ }^{19}$ If there was no evi- dence against the homogeneity of odds ratios, the Mantel-Haenszel estimate of the common odds ratio was calculated in addition to the gross odds ratio. ${ }^{20}$ All statistical analyses were performed with the use of SAS software, version 9.2, and the $\mathrm{R}$ statistical package, version 2.11.

\section{RESULTS}

\section{CHARACTERISTICS OF THE PATIENTS AND THE TUMORS}

Between January 5, 2007, and June 30, 2010, a total of 1206 patients were enrolled (Fig. S1 in the Supplementary Appendix). The characteristics of the patients and the tumors were well balanced across the treatment groups (Table 1, and Table S1 in the Supplementary Appendix). A total of $47 \%$ of the patients had clinically positive nodes, $55 \%$ of the tumors were poorly differentiated, and $59 \%$ of the tumors were hormone-receptor-positive. The hormone-receptor status was assessed at the participating institutions according to their own criteria for positivity.

The primary analyses were performed on data from all patients who underwent randomization and for whom outcomes were ascertained. Assessments of the primary end point, pathological complete response in the breast, were available for 1186 of the 1206 patients who underwent randomization; among the 20 patients who were not included in the primary analyses, 13 withdrew consent and 7 had missing data. Two of the 1186 patients had inoperable disease after chemotherapy.

An additional 20 patients (1.7\%), with a balanced distribution across the treatment groups, were found after randomization to be ineligible: 6 did not have preentry measurements of the urinary protein:creatinine ratio, 3 had stage T4 tumors, 2 had HER2-positive cancer, and 9 had various other reasons. The secondary analyses, including subgroup analyses, were performed on data from the 1166 eligible patients.

\section{EFFECTS OF ADDING CAPECITABINE OR GEMCITABINE}

The addition of capecitabine or gemcitabine did not increase the rate of pathological complete response in the breast $(\mathrm{P}=0.26$, with adjustment for multiple comparisons according to the Dunnett method). There were no significant differences among the three chemotherapy regimens with respect to 


\begin{tabular}{|c|c|c|c|c|c|c|c|}
\hline Characteristic & Docetaxel & $\begin{array}{l}\text { Docetaxel plus } \\
\text { Bevacizumab }\end{array}$ & $\begin{array}{l}\text { Docetaxel- } \\
\text { Capecitabine }\end{array}$ & $\begin{array}{c}\text { Docetaxel- } \\
\text { Capecitabine plus } \\
\text { Bevacizumab }\end{array}$ & $\begin{array}{l}\text { Docetaxel- } \\
\text { Gemcitabine }\end{array}$ & $\begin{array}{c}\text { Docetaxel- } \\
\text { Gemcitabine plus } \\
\text { Bevacizumab }\end{array}$ & $P$ Value \\
\hline Total cohort (no.) & 201 & 199 & 204 & 201 & 197 & 204 & \\
\hline Ineligible patients (no.) & 1 & 4 & 4 & 4 & 5 & 2 & 0.88 \\
\hline Age at entry (\%) & & & & & & & 0.98 \\
\hline$\leq 49 \mathrm{yr}$ & 54 & 52 & 50 & 53 & 52 & 52 & \\
\hline $50-59 \mathrm{yr}$ & 31 & 33 & 32 & 28 & 34 & 31 & \\
\hline$\geq 60 \mathrm{yr}$ & 14 & 15 & 18 & 19 & 14 & 17 & \\
\hline Clinical tumor size (\%) & & & & & & & 0.98 \\
\hline $2-4 \mathrm{~cm}$ & 44 & 43 & 48 & 47 & 45 & 46 & \\
\hline$>4 \mathrm{~cm}$ & 56 & 57 & 52 & 53 & 55 & 54 & \\
\hline Clinical nodal status (\%) & & & & & & & 1.00 \\
\hline Positive & 48 & 47 & 46 & 46 & 46 & 46 & \\
\hline Negative & 52 & 54 & 54 & 54 & 54 & 54 & \\
\hline Hormone-receptor status (\%) & & & & & & & 1.00 \\
\hline Positive & 60 & 60 & 58 & 59 & 59 & 60 & \\
\hline Negative & 40 & 40 & 42 & 41 & 41 & 40 & \\
\hline Race (\%)† & & & & & & & 0.06 \\
\hline White & 84 & 87 & 80 & 87 & 76 & 86 & \\
\hline Black & 12 & 10 & 17 & 10 & 17 & 14 & \\
\hline Other & 3 & 3 & 3 & 3 & 7 & $<1$ & \\
\hline Histologic grade of tumor (\%) & & & & & & & 0.99 \\
\hline Low & 7 & 6 & 7 & 8 & 8 & 6 & \\
\hline Intermediate & 36 & 35 & 33 & 38 & 33 & 38 & \\
\hline High & 55 & 57 & 58 & 53 & 56 & 53 & \\
\hline Unknown & 1 & 2 & 2 & $<1$ & 3 & 2 & \\
\hline
\end{tabular}

* Patients were randomly assigned to receive docetaxel, docetaxel-capecitabine, or docetaxel-gemcitabine for four cycles, followed in all groups by four cycles of doxorubicin-cyclophosphamide. In addition, half the patients were also randomly assigned to receive bevacizumab with each of the first six cycles of chemotherapy.

$\uparrow$ Race was self-reported.

the rate of pathological complete response in the breast or the rate of pathological complete response in the breast and nodes (rates of pathological complete response in the breast, $32.7 \%$ with docetaxel, $29.7 \%$ with docetaxel-capecitabine, and 31.8\% with docetaxel-gemcitabine; $\mathrm{P}=0.69$ with the use of Pearson's chi-square test with continuity adjustment; rates of pathological complete response in the breast and nodes, $25.8 \%, 23.2 \%$, and $26.9 \%$, in the three groups, respectively; $\mathrm{P}=0.51$ ) (Fig. 1). The results for the 1166 eligible patients were similar $(\mathrm{P}=0.76$ and $\mathrm{P}=0.53$ for the comparisons of pathological complete response in the breast and in the breast and nodes, respectively). Subset anal- yses according to clinical tumor size, clinical nodal status, hormone-receptor status, age, and tumor grade did not show significant differences among the chemotherapy regimens in any subgroup of patients (Fig. S2 in the Supplementary Appendix).

Clinical responses were assessed after completion of the docetaxel-based cycles in the case of 1155 patients and before surgery in the case of 1163. The rates of clinical complete response in the three chemotherapy groups were similar after the four docetaxel-based cycles $(36.6 \%$ with docetaxel, $32.3 \%$ with docetaxel-capecitabine, and $40.2 \%$ with docetaxel-gemcitabine; $\mathrm{P}=0.09$ ) and after completion of all chemotherapy before surgery 


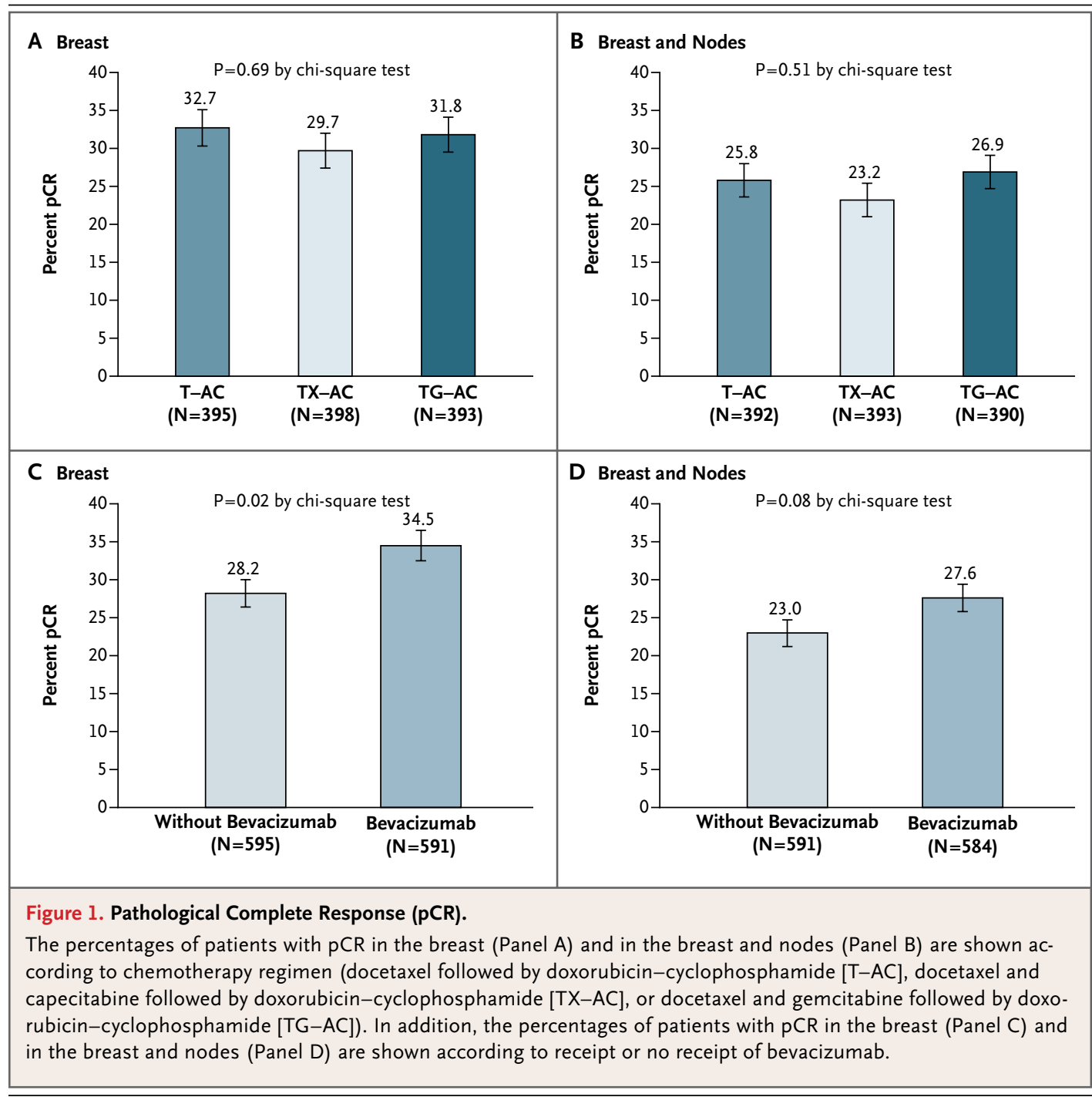

(58.3\%, 55.4\%, and 56\%, respectively; $\mathrm{P}=0.74$ ). The rates of clinical partial response were also similar (Table S2 in the Supplementary Appendix). The proportions of patients who underwent breastconserving surgery were similar in the three groups (46\%, $43 \%$, and 50\%, respectively).

\section{EFFECTS OF ADDING BEVACIZUMAB}

The addition of bevacizumab significantly increased the rate of pathological complete response in the breast, from $28.2 \%$ to $34.5 \%(\mathrm{P}=0.02)$ (Fig. 1C). When the rate of pathological complete response was examined according to hormone-receptor status, the effect of bevacizumab was more pronounced in the hormone-receptor-positive subset $(15.1 \%$ without bevacizumab vs. $23.2 \%$ with bevacizumab, $\mathrm{P}=0.007)$, with a weaker effect in the hormone-receptor-negative subset $(47.1 \%$ without bevacizumab vs. $51.5 \%$ with bevacizumab, $\mathrm{P}=0.34$ ). The effects of adding bevacizumab on the rates of pathological complete response in the 1166 eligible patients were similar to those in the primaryanalysis cohort $(\mathrm{P}=0.02$ for the effect in the overall cohort, $\mathrm{P}=0.005$ for the effect in the hormonereceptor-positive subset, and $\mathrm{P}=0.29$ for the effect in the hormone-receptor-negative subset). However, the Breslow-Day test for homogeneity of odds ratios across hormone-receptor subsets was not significant $(\mathrm{P}=0.19)$ (Fig. 2A). The rate of pathological complete response in the breast was significantly increased when bevacizumab was added to the docetaxel-capecitabine regimen $(36.1 \%$ vs. 


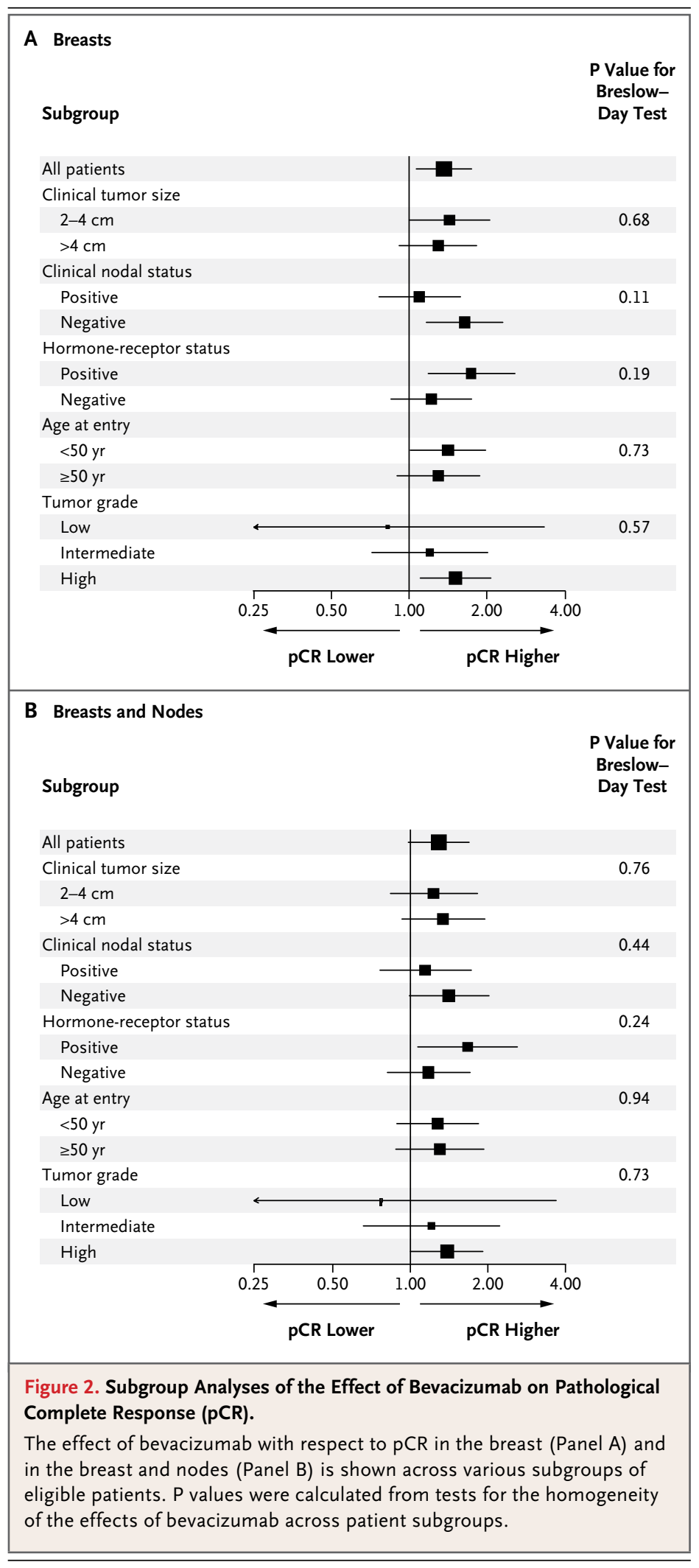

$23.5 \%, \mathrm{P}=0.009)$ but not when it was added to the docetaxel-gemcitabine regimen (35.8\% vs. $27.6 \%$, $\mathrm{P}=0.10$ ) or the docetaxel regimen ( $31.6 \%$ vs. $33.7 \%$, $\mathrm{P}=0.75$ ). The $\mathrm{P}$ value for the test for homogeneity of the odds ratios according to chemotherapy regimen was 0.07 . The results from the multiple logistic-regression model showed that hormonereceptor-negative status, high tumor grade, and smaller tumor size were associated with higher rates of pathological complete response in the breast (Table S3 in the Supplementary Appendix).

There was an increase in the rate of pathological complete response in the breast and nodes with bevacizumab therapy, but the difference in the overall cohort was not significant $(23.0 \%$ without bevacizumab vs. $27.6 \%$ with bevacizumab, $\mathrm{P}=0.08$ ) (Fig. 1D); however, in the hormone-receptor-positive subset, the increase with the addition of bevacizumab therapy did reach significance $(11.1 \%$ without bevacizumab vs. $16.8 \%$ with bevacizumab, $\mathrm{P}=0.03$ ). Again, however, the test for homogeneity of the odds ratios was not significant $(\mathrm{P}=0.25)$. The results in eligible patients were similar to those in the primary-analysis cohort (Fig. 2B). There was also a trend toward a greater effect of bevacizumab with an increase in tumor grade, but the test for homogeneity of the odds ratios was not significant (Fig. 2B).

The addition of bevacizumab increased the rate of clinical complete response, with a rate of $51.7 \%$ among patients who did not receive bevacizumab, as compared with $61.5 \%$ among those who received bevacizumab $(\mathrm{P}<0.001)$. All classes of clinical responses, in patients who received bevacizumab and in patients who did not receive bevacizumab, are shown in Table S4 in the Supplementary Appendix. The effect of bevacizumab on clinical complete response was similar to the effect on pathological complete response, with a more pronounced effect in the hormone-receptorpositive subset $(50.7 \%$ without bevacizumab vs. $62.2 \%$ with bevacizumab, $\mathrm{P}=0.003$ ) than in the hormone-receptor-negative subset (53.1 without bevacizumab vs. $60.4 \%$ with bevacizumab, $\mathrm{P}=0.14$ ).

\section{TOXIC EFFECTS}

Table S5 in the Supplementary Appendix shows the percentages of patients who completed all planned neoadjuvant treatments and the reasons for discontinuation for those who did not. A total of $83 \%$ of the patients who did not receive beva- 


\begin{tabular}{|c|c|c|c|c|c|c|c|c|c|c|}
\hline \multirow[t]{2}{*}{ Toxic Effect } & \multicolumn{3}{|c|}{$\begin{array}{l}\text { Docetaxel } \\
(\mathrm{N}=396)\end{array}$} & \multicolumn{3}{|c|}{$\begin{array}{l}\text { Docetaxel-Capecitabine } \\
\qquad(\mathrm{N}=399)\end{array}$} & \multicolumn{3}{|c|}{$\begin{array}{l}\text { Docetaxel-Gemcitabine } \\
\qquad(\mathrm{N}=396)\end{array}$} & \multirow[t]{3}{*}{ P Value } \\
\hline & Grade 2 & Grade 3 & Grade 4 & Grade 2 & Grade 3 & Grade 4 & Grade 2 & Grade 3 & Grade 4 & \\
\hline \multicolumn{10}{|c|}{ percent of patients } & \\
\hline Overall $†$ & 16 & 48 & 7 & 20 & 56 & 13 & 14 & 61 & 11 & $<0.001$ \\
\hline Leukopenia & 0 & 5 & $<1$ & 0 & 4 & 1 & 0 & 9 & 1 & 0.17 \\
\hline Neutropenia & 0 & 10 & 5 & 0 & 13 & 7 & 0 & 26 & 8 & $<0.001$ \\
\hline Hypertension & 7 & 6 & $<1$ & 7 & 5 & $<1$ & 9 & 5 & 0 & 0.94 \\
\hline $\begin{array}{l}\text { Left ventricular systolic } \\
\text { dysfunction }\end{array}$ & 2 & 0 & 0 & $<1$ & 2 & 0 & 1 & $<1$ & $<1$ & 0.43 \\
\hline Fatigue & 0 & 9 & 0 & 0 & 10 & $<1$ & 0 & 9 & 0 & 0.97 \\
\hline Hand-foot syndrome & 9 & 3 & 0 & 28 & 23 & 0 & 3 & 1 & 0 & $<0.001$ \\
\hline Rash & 11 & $<1$ & $<1$ & 9 & 1 & $<1$ & 17 & $<1$ & 0 & 0.03 \\
\hline Diarrhea & 10 & 3 & 0 & 16 & 8 & $<1$ & 14 & 7 & 0 & 0.02 \\
\hline Mucositis & 8 & 2 & 0 & 16 & 4 & $<1$ & 13 & 3 & 0 & 0.01 \\
\hline Nausea & 0 & 3 & 0 & 0 & 5 & 0 & 0 & 5 & $<1$ & 0.91 \\
\hline Vomiting & 0 & 2 & 0 & 0 & 4 & 0 & 0 & 6 & $<1$ & 0.46 \\
\hline Febrile neutropenia & 0 & 6 & 0 & 0 & 10 & $<1$ & 0 & 8 & $<1$ & 0.61 \\
\hline Infection in wound & 0 & 1 & 0 & 0 & 1 & 0 & 0 & 2 & 0 & 0.83 \\
\hline Sensory neuropathy & 13 & 4 & 0 & 13 & 4 & 0 & 8 & 2 & 0 & 0.04 \\
\hline Bone pain & 0 & 6 & $<1$ & 0 & 2 & 0 & 0 & 3 & 0 & 0.12 \\
\hline Headache & 0 & 2 & $<1$ & 0 & 2 & $<1$ & 0 & 3 & 0 & 0.95 \\
\hline Dyspnea & 5 & $<1$ & 0 & 2 & 2 & $<1$ & 5 & 1 & $<1$ & 0.40 \\
\hline $\begin{array}{l}\text { Thrombosis, thrombus, } \\
\text { or embolism }\end{array}$ & $<1$ & $<1$ & $<1$ & 1 & 2 & 2 & 1 & 2 & $<1$ & 0.73 \\
\hline
\end{tabular}

* Patients in all three groups were randomly assigned to receive four cycles of the assigned therapy, followed by four cycles of doxorubicincyclophosphamide.

$\dagger$ The rate of death in the overall cohort during the treatment period was less than $1 \%$ in the docetaxel and docetaxel-capecitabine groups and 0 in the docetaxel-gemcitabine group.

cizumab, as compared with $78 \%$ of those who received bevacizumab, completed all planned preoperative therapy. Tables 2 and 3 list the overall incidences of toxic effects, toxic effects (grades 2,3 , or 4 ) occurring in more than $5 \%$ of the patients, treatment-related deaths, and other serious toxic effects in the three chemotherapy groups (Table 2) and in all patients according to whether they received or did not receive bevacizumab (Table 3). The addition of capecitabine or gemcitabine increased the rate of overall toxic effects. In the docetaxel-capecitabine group, the increased rate of toxic effects was largely the result of increases in grades 2 and 3 hand-foot syndrome. In the docetaxel-gemcitabine group, the toxic effect with the greatest increase in frequency, as compared with the toxic effects seen with docetaxel alone, was neutropenia.

The addition of bevacizumab to chemotherapy also increased the toxic effects, particularly the rates of hypertension, mucositis, and the handfoot syndrome (Table 3). The patients who received bevacizumab also had a significant increase in left ventricular dysfunction. A total of 8 patients $(1.3 \%)$ who received bevacizumab, as compared with 1 patient $(0.2 \%)$ who did not receive bevacizumab, had left ventricular dysfunction of grade 3 or grade 4 (with the one case of grade 4 left ventricular dysfunction in the bevacizumab group); the majority of cases were grade 2 (11 in the group 


\begin{tabular}{|c|c|c|c|c|c|c|c|}
\hline \multirow[t]{2}{*}{ Toxic Effect } & \multicolumn{3}{|c|}{ No Bevacizumab ( $N=596$ ) } & \multicolumn{3}{|c|}{ Bevacizumab ( $N=595)$} & \multirow[t]{3}{*}{ P Value } \\
\hline & Grade 2 & Grade 3 & Grade 4 & Grade 2 & Grade 3 & Grade 4 & \\
\hline \multicolumn{7}{|c|}{ percent of patients } & \\
\hline Overall $\uparrow$ & 18 & 49 & 9 & 15 & 61 & 12 & $<0.001$ \\
\hline Leukopenia & 0 & 6 & 1 & 0 & 6 & $<1$ & 0.47 \\
\hline Neutropenia & 0 & 16 & 6 & 0 & 16 & 7 & 1.00 \\
\hline Hypertension & 1 & $<1$ & 0 & 13 & 10 & $<1$ & $<0.001$ \\
\hline Left ventricular systolic dysfunction & $<1$ & $<1$ & 0 & 2 & 1 & $<1$ & 0.02 \\
\hline Fatigue & 0 & 9 & $<1$ & 0 & 10 & $<1$ & 0.95 \\
\hline Hand-foot syndrome & 11 & 7 & 0 & 15 & 11 & 0 & 0.008 \\
\hline Rash & 10 & $<1$ & $<1$ & 14 & 1 & $<1$ & 0.34 \\
\hline Diarrhea & 13 & 7 & $<1$ & 14 & 5 & 0 & 0.67 \\
\hline Mucositis & 11 & 1 & 0 & 14 & 4 & $<1$ & 0.007 \\
\hline Nausea & 0 & 4 & $<1$ & 0 & 5 & 0 & 0.99 \\
\hline Vomiting & 0 & 5 & $<1$ & 0 & 3 & 0 & 0.45 \\
\hline Febrile neutropenia & 0 & 6 & $<1$ & 0 & 10 & $<1$ & 0.15 \\
\hline Infection in wound & 0 & $<1$ & 0 & 0 & 3 & 0 & 1.00 \\
\hline Sensory neuropathy & 11 & 3 & 0 & 12 & 4 & 0 & 0.55 \\
\hline Bone pain & 0 & 3 & $<1$ & 0 & 4 & 0 & 0.39 \\
\hline Headache & 0 & $<1$ & 0 & 0 & 4 & $<1$ & 0.01 \\
\hline Dyspnea & 4 & $<1$ & 0 & 4 & 1 & $<1$ & 0.69 \\
\hline Thrombosis, thrombus, or embolism & $<1$ & 1 & $<1$ & 1 & 2 & 1 & 0.78 \\
\hline
\end{tabular}

* Patients were randomly assigned to receive bevacizumab with each of the first six cycles of chemotherapy. $\uparrow$ The rate of death in the overall cohort during the treatment period was less than $1 \%$ in both groups.

that received bevacizumab vs. 1 in the group that did not receive bevacizumab) (Table 3). A more detailed analysis of the effect on left ventricular function of adding bevacizumab is provided in Table S6 in the Supplementary Appendix. There was a low incidence of wound complications in the group that received bevacizumab, but the rate was significantly higher than the rate in the group that did not receive bevacizumab ( $2 \%$ vs. $0 \%$, $\mathrm{P}=0.002)$.

\section{DISCUSSION}

In this trial, neither capecitabine nor gemcitabine added to an anthracycline-taxane based chemotherapy regimen improved the rates of clinical or pathological response, despite the suggestive results that have been seen in patients with metastatic breast cancer. Thus, it appears unlikely that these drugs would add much benefit in the adjuvant setting. Indeed, our results confirm (and would have predicted) the largely negative results of several trials of adjuvant therapy with these drugs, ${ }^{21-23}$ supporting our hypothesis that trials of neoadjuvant therapy serve as better predictors of adjuvant benefits than studies of metastatic disease. The addition of bevacizumab resulted in a modest but significant increase in the rate of pathological complete response in the breast, but the rate of pathological complete response in the breast and nodes was not significantly increased, which may indicate that this drug will have a lesser effect on patient outcomes. Moreover, adding bevacizumab increased a number of toxic effects. Left ventricular dysfunction was noted as a serious concern in a recent meta-analysis of bevacizumab therapy in patients with metastatic breast cancer. ${ }^{24}$ Left ventricular function and wound complications are being closely monitored in patients receiving adjuvant bevacizumab therapy as well as in the long-term 
follow-up of these patients. It is unclear why the greatest benefit from adding an antiangiogenic agent was seen in patients with hormone-receptorpositive tumors, in contrast to the findings in the GeparQuinto trial (NCT00567554), reported by von Minckwitz et al. elsewhere in this issue of the Journal, ${ }^{25}$ in which the benefit was confined to patients with hormone-receptor-negative tumors. The disparity in the results of the two trials may be related to differences in the inclusion criteria and the study design, particularly the inclusion in the GeparQuinto trial of patients with more advanced disease, a different sequencing of drug regimens in the GeparQuinto trial than that in our trial, and the withdrawal from the GeparQuinto study of patients who did not have a response to the initial four cycles of treatment. ${ }^{25}$

The benefit of bevacizumab in our study also tended to be seen in patients with a high tumor grade (Fig. 2A and 2B), a finding that was also observed in the GeparQuinto study. The increased rate of pathological complete response in patients with hormone-receptor-positive tumors is encouraging, since this group tends to have low rates of pathological complete response with chemotherapy. The addition of an antimetabolite (capecitabine or gemcitabine) in two thirds of our patients, with a concomitant decrease in the dose of docetaxel, might account for the disproportionate effect of adding bevacizumab in the docetaxel-capecitabine and docetaxel-gemcitabine groups. The effect of adding bevacizumab in the NSABP B-40 trial was less dramatic than was the effect of adding docetaxel in the NSABP B-27 trial, so it is not clear whether the neoadjuvant effect of bevacizumab would translate into a substantial benefit to patients. However, the groups that were randomly assigned to bevacizumab in our trial also received bevacizumab postoperatively, so the potential for bevacizumab to improve the outcomes should be clarified when the results with respect to diseasefree survival and overall survival are available from the NSABP B-40 trial and from studies of adjuvant bevacizumab therapy that are currently in progress. In addition, the collection of tissue samples from all our patients before treatment, a major advantage of the neoadjuvant approach, offers an opportunity to discover molecular markers that might predict a benefit from bevacizumab. Such markers would allow the selective use of this agent in the subsets of patients most likely to have improved outcomes with the use of the drug.

Presented in part at the American Society of Clinical Oncology Symposium, Chicago, June 3-7, 2011.

Supported by grants from the National Cancer Institute (U10CA-12027, U10-CA-69651, U10-CA-37377, U10-CA-69974, and U10-CA-44066), the Department of Health and Human Services, the Public Health Service, F. Hoffmann-La Roche, Genentech USA, and Eli Lilly.

Disclosure forms provided by the authors are available with the full text of this article at NEJM.org.

We thank Barbara C. Good, Ph.D., Wendy L. Rea, and Christine I. Rudock of the National Surgical Adjuvant Breast and Bowel Project for editorial assistance.
REFERENCES

1. Fisher B, Brown A, Mamounas E, et al. Effect of preoperative chemotherapy on local-regional disease in women with operable breast cancer: findings from $\mathrm{Na}$ tional Surgical Adjuvant Breast and Bowe Project B-18. J Clin Oncol 1997;15:2483-93. 2. Fisher B, Bryant J, Wolmark N, et al. Effect of preoperative chemotherapy on the outcome of women with operable breast cancer. J Clin Oncol 1998;16:2672-85.

3. Gianni L, Baselga J, Eiermann W, et al. Phase III trial evaluating the addition of paclitaxel to doxorubicin followed by cyclophosphamide, methotrexate, and fluorouracil, as adjuvant or primary systemic therapy: European Cooperative Trial in Operable Breast Cancer. J Clin Oncol 2009; 27:2474-81.

4. Mamounas EP, Brown A, Anderson S, et al. Sentinel node biopsy after neoadjuvant chemotherapy in breast cancer: results from National Surgical Adjuvant Breast and Bowel Project Protocol B-27. J Clin Oncol 2005;23:2694-702. [Erratum, J Clin Oncol 2005;23:4808.]
5. Bear HD, Anderson S, Brown A, et al. The effect on tumor response of adding sequential preoperative docetaxel to preoperative doxorubicin and cyclophosphamide: preliminary results from National Surgical Adjuvant Breast and Bowel Project Protocol B-27. J Clin Oncol 2003;21: 4165-74.

6. Hunt KK, Yi M, Mittendorf EA, et al. Sentinel lymph node surgery after neoadjuvant chemotherapy is accurate and reduces the need for axillary dissection in breast cancer patients. Ann Surg 2009; 250:558-66.

7. Bear HD, Anderson S, Smith RE, et al. Sequential preoperative or postoperative docetaxel added to preoperative doxorubicin plus cyclophosphamide for operable breast cancer: National Surgical Adjuvant Breast and Bowel Project Protocol B-27. J Clin Oncol 2006;24:2019-27.

8. Henderson IC, Berry DA, Demetri GD, et al. Improved outcomes from adding sequential paclitaxel but not from escalating doxorubicin dose in an adjuvant chemo- therapy regimen for patients with nodepositive primary breast cancer. J Clin Oncol 2003;21:976-83.

9. Mamounas EP, Bryant J, Lembersky B, et al. Paclitaxel after doxorubicin plus cyclophosphamide as adjuvant chemotherapy for node-positive breast cancer: results from NSABP B-28. J Clin Oncol 2005;23: 3686-96.

10. Martin M, Pienkowski T, Mackey J, et al. Adjuvant docetaxel for node-positive breast cancer. N Engl J Med 2005;352:230213.

11. Roché H, Fumoleau P, Spielmann M, et al. Sequential adjuvant epirubicin-based and docetaxel chemotherapy for nodepositive breast cancer patients: the FNCLCC PACS 01 Trial. J Clin Oncol 2006;24:566471.

12. Miller K, Wang M, Gralow J, et al. Paclitaxel plus bevacizumab versus paclitaxel alone for metastatic breast cancer. $\mathrm{N}$ Engl J Med 2007;357:2666-76.

13. Robert NJ, Diéras V, Glaspy J, et al. RIBBON-1: randomized, double-blind, pla- 
cebo-controlled, phase III trial of chemotherapy with or without bevacizumab for first-line treatment of human epidermal growth factor receptor 2-negative, locally recurrent or metastatic breast cancer. J Clin Oncol 2011;29:1252-60.

14. Miles DW, Chan A, Dirix LY, et al. Phase III study of bevacizumab plus docetaxel compared with placebo plus docetaxel for the first-line treatment of human epidermal growth factor receptor 2-negative metastatic breast cancer. J Clin Oncol 2010;28:3239-47.

15. O'Shaughnessy J, Miles D, Vukelja S, et al. Superior survival with capecitabine plus docetaxel combination therapy in anthracycline-pretreated patients with advanced breast cancer: phase III trial results. J Clin Oncol 2002;20:2812-23.

16. Albain KS, Nag SM, Calderillo-Ruiz $\mathrm{G}$, et al. Gemcitabine plus paclitaxel versus paclitaxel monotherapy in patients with metastatic breast cancer and prior anthracycline treatment. J Clin Oncol 2008; 26:3950-7.
17. Therasse P, Arbuck SG, Eisenhauer EA, et al. New guidelines to evaluate the response to treatment in solid tumors J Natl Cancer Inst 2000;92:205-16.

18. Dunnett CW. A multiple comparison procedure for comparing several treatments with a control. J Am Stat Assoc 1955;50:1096-121.

19. Breslow NE, Day NE. Statistical methods in cancer research. Vol. 1. The analysis of case-control studies. Lyon, France: International Agency for Research on Cancer, 1980. (IARC scientific publication no. 32.)

20. Mantel N, Haenszel W. Statistical aspects of the analysis of data from retrospective studies of disease. J Natl Cancer Inst 1959;22:719-48.

21. Joensuu H, Kellokumpu-Lehtinen P-L, Huovinen R, et al. FinXX final 5-year analysis: results of the randomized, openlabel phase III trial in medium-to-high risk early breast cancer. Cancer Res 2010; 70:Suppl 2:84s. abstract.

22. O'Shaughnessy J, Paul D, Stokoe C, et al. First efficacy results of a randomized, open-label, phase III study of adjuvant doxorubicin plus cyclophosphamide, followed by docetaxel with or without capecitabine, in high-risk early breast cancer. Cancer Res 2010;70:Suppl 2:85s. abstract.

23. Poole CJ, Hiller L, Howard HC, et al. tAnGo: a randomized phase III trial of gemcitabine (gem) in paclitaxel-containing, epirubicin/cyclophosphamide-based, adjuvant chemotherapy (CT) for women with early-stage breast cancer (EBC). J Clin Oncol 2008;26:Suppl:8s. abstract.

24. Verma N, Swain SM. Bevacizumab and heart failure risk in patients with breast cancer: a thorn in the side? J Clin Oncol 2011;29:603-6.

25. von Minckwitz G, Eidtmann H, Rezai $M$, et al. Neoadjuvant chemotherapy and bevacizumab for HER2-negative breast cancer. N Engl J Med 2012;366:299-309.

Copyright (c) 2012 Massachusetts Medical Society.

The NEJM Image Challenge app brings a popular online feature to the smartphone. Optimized for viewing on the iPhone and iPod Touch, the Image Challenge app lets you test your diagnostic skills anytime, anywhere. The Image Challenge app randomly selects from 300 challenging clinical photos published in NEJM, with a new image added each week. View an image, choose your answer, get immediate feedback, and see how others answered.

The Image Challenge app is available at the iTunes App Store. 